\title{
Dynamic configurational anisotropy in nanomagnets
}

\author{
V. V. Kruglyak, P. S. Keatley, and R. J. Hicken \\ School of Physics, University of Exeter, Stocker Road, Exeter EX4 4QL, United Kingdom \\ J. R. Childress and J. A. Katine \\ Hitachi Global Storage Technologies, San Jose Research Center, 650 Harry Road, San Jose, California 95120, USA
}

(Received 9 November 2006; published 5 January 2007)

\begin{abstract}
The angular dependence of ultrafast magnetization dynamics in nanomagnets of square shape was studied by magneto-optical pump-probe measurements. In agreement with micromagnetic simulations, both the number of precessional modes and the values of their frequencies were observed to vary as the orientation of the external magnetic field was rotated in the element plane. We show that the observed behavior cannot be explained by the angular variation of the static effective magnetic field. Instead, it is found to originate from a new type of magnetic anisotropy - a dynamic configurational anisotropy, which is due to the variation of the dynamic effective magnetic field. Although always present, the dynamical anisotropy may dominate in nanoscale magnetic elements in which the static configurational anisotropy is suppressed.
\end{abstract}

DOI: 10.1103/PhysRevB.75.024407 PACS number(s): 75.30.Gw, 75.40.Gb, 75.75.+a, 75.30.Ds

\section{INTRODUCTION}

According to Noether's theorem, ${ }^{1}$ there is a fundamental connection between the symmetry of a physical system and the conservation laws governing its behavior. For instance, time invariance implies that energy is conserved, while translational invariance implies that linear momentum is conserved. Invariance with respect to rotation (so-called isotropy) leads to conservation of angular momentum. The spin and orbital electronic angular momentum are responsible for the magnetic properties of solids. Consequently any violation of the invariance with respect to rotation of the magnetization direction (called magnetic anisotropy ${ }^{2,3}$ ) is of crucial importance for understanding both the fundamental properties of magnetic systems and their applications, e.g., in magnetic data storage technology.

The internal magnetic field in elongated or flattened ferromagnetic elements is different when the external magnetic field is applied along the long and short axes. ${ }^{2,3}$ This effect is sometimes referred to as the shape anisotropy. Generally, the shape anisotropy is determined by the aspect ratio of the element and exists irrespective of the uniformity of the magnetic configuration. In particular, shape anisotropy occurs in ellipsoidal magnetic elements in which the internal magnetic field and hence the magnetization are uniform. However, a simple calculation shows that the internal magnetic field in the center of a thin square uniformly magnetized element would not depend upon the direction of the external magnetic field used to align the magnetization within the plane of the element.

In magnetic nanoelements with a nonellipsoidal shape, small deviations of the static magnetization from the uniformly magnetized state result in a strong anisotropy with a symmetry that, to a first approximation, corresponds to that of the shape of the nanoelement. ${ }^{4-7}$ Schabes and Bertram identified this so-called configurational anisotropy from a numerical study of the dependence of the magnetic hysteresis loop upon the direction of the magnetic field applied to ferromagnetic nanocubes. ${ }^{4}$ Cowburn et al. studied experimentally the dependence of the total free energy of and the average total effective magnetic field in thin nanoelements upon the direction of a bias magnetic field of constant magnitude ${ }^{6,7}$ They showed that the effective magnetic field due to the configurational anisotropy could have a magnitude of a few hundred Oersteds in the center of a magnetic nanoelement and so could easily dominate its static magnetic properties.

The manifestations of the configurational anisotropy in the magnetization dynamics of magnetic elements were discussed further in a series of recent publications by different groups. Chérif et al. observed a difference between spin wave frequencies within the Brillouin light scattering (BLS) spectra measured with the bias magnetic field applied parallel to the edge and to the diagonal of rectangular elements. ${ }^{8,9}$ The effect of the shape and configurational anisotropies upon the ferromagnetic resonance (FMR) mode was studied by Zhai et al. ${ }^{10}$ and more recently by Pardavi-Horvath et al. ${ }^{11}$ The spatial character of the anisotropic magnetization dynamics in micron sized square magnetic elements was directly studied by time resolved scanning Kerr microscopy (TRSKM) by Barman et al. ${ }^{12-15}$ In addition to the fourfold variation of the frequency of the uniform precessional mode, they found that the spatial character of magnetostatic modes of finite wave number depends sensitively upon the direction of the bias magnetic field, and the dephasing of the modes leads to an anisotropic apparent damping of the precessional signal.

In contrast to the isolated element anisotropy discussed above, which can be referred to as an intrinsic configurational anisotropy, the effective magnetic field inside an element within an array can also contain an anisotropic contribution due to the stray field from the neighboring elements. ${ }^{16-20}$ This anisotropy is mediated by the magnetically unsaturated regions situated near the edges of the elements, ${ }^{16}$ and can be referred to as an extrinsic configurational anisotropy.

The anisotropic variation of the high frequency magnetization dynamics observed in Refs. 8-20 could be explained by an anisotropic modification of the static effective magnetic field in the magnetic element either due to the configu- 
ration of the static magnetization or due to the static stray field from the neighbors. However, Cowburn et al. found that the static configurational anisotropy essentially vanishes in squares of thickness equal to or smaller than $3 \mathrm{~nm}$ and of a size equal to or smaller than $200 \mathrm{~nm}^{7}$ Here, we report an angular variation of the precessional mode frequencies of a square magnetic nanoelement of similar size and thickness. The variation of frequency with bias field orientation is governed in this regime by the variation of both the static and dynamic magnetization configuration and the associated dynamic effective magnetic field. There is therefore a dynamic configurational anisotropy. The anisotropy is intrinsic and originates from the anisotropy of the magnetodipole interaction, which has also been known to govern the dispersion of magnetostatic modes in uniformly magnetized magnetic slabs $^{21}$ and in magnetic stripes with nonuniform magnetization. $^{22}$

Specifically, we study the magnetization dynamics in a $4 \times 4 \mu \mathrm{m}^{2}$ array of $220 \times 220 \times 2.5 \mathrm{~nm}^{3}$ square ferromagnetic elements ${ }^{23,24}$ with a $95 \mathrm{~nm}$ edge-to-edge separation by means of magneto-optical pump-probe measurements and micromagnetic simulations. The angular variation of the magnetization dynamics in elements of a different size, e.g., those in Refs. 23 and 24, will in general be governed by a competition of dynamic and static configurational anisotropies. The time dependent response of the out-of-plane component of the magnetization to a pulsed magnetic field was measured via the polar Kerr effect by focusing a time delayed optical probe pulse to a submicron spot in the center of the array. The size of an individual element was smaller than the spatial resolution of the experiment. Therefore, numerical simulations performed with the Object Oriented Micromagnetic Framework (OOMMF) (Ref. 25) were used instead to reproduce the observed variations in the mode frequencies, and, once the agreement between the experiment and the simulations was established, to map the spatial distributions of the out-of-plane component of the dynamic magnetization and to calculate the associated effective fields. ${ }^{26}$ The details of the experimental setup, the magnetic parameters of the sample, and the methodology of the micromagnetic simulations can be found in Refs. 23, 24, and 26.

\section{EXPERIMENTAL AND NUMERICAL RESULTS}

The measured time resolved (TR) Kerr signals and their fast Fourier transform (FFT) spectra are presented in Figs. 1 and 2 for different values and orientations of the bias magnetic field. In Fig. 1, when the field is applied parallel to an element edge, the FFT spectrum contains two modes. In Ref. 24 , the higher and the lower frequency modes were found to be associated with the central area of the element ("center mode") and the element edges perpendicular to the field direction ("edge mode"), respectively. The edge mode FFT peaks are noticeably broader than those of the center mode, which was interpreted in Ref. 24 in terms of the increased influence of the edge roughness. For the field applied parallel to a diagonal of the element, the FFT spectrum contains only one mode at a frequency that is similar to but slightly smaller than that of the center mode observed when the field was applied parallel to an edge.

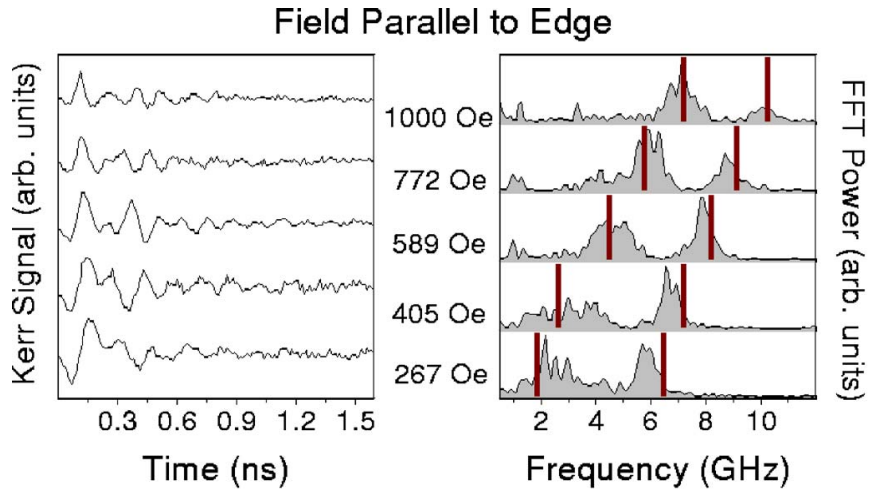

Field Parallel to Diagonal

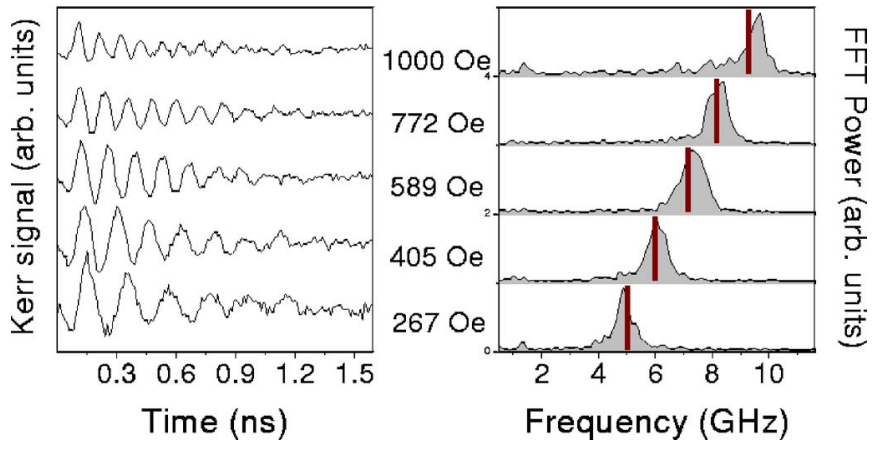

FIG. 1. (Color online) TR Kerr signals (left panels) and their FFT spectra (right panels) obtained for different values of the bias magnetic field are shown for the field applied parallel to the edge (upper panels) and diagonal (lower panels) of the element. In each panel, curves from top to bottom correspond to bias field values of 1000, 772, 589, 405, and 267 Oe, respectively. The vertical dashes show the frequencies of the modes obtained from the micromagnetic simulations.

In order to elucidate how the transition between the "two mode" and "single mode" regimes occurs, measurements were performed as a bias field of fixed value (589 Oe) was rotated through $360^{\circ}$ in steps of $15^{\circ}$. The results are shown in Fig. 2. In the FFT spectra obtained with the field aligned $15^{\circ}$ from the element edges, a strong peak was observed at a frequency somewhere in between those of the edge and center modes observed with the field parallel to an edge. A smaller, less distinct peak was also sometimes observed at a frequency higher than that of the center mode observed with the field parallel to an edge. In the FFT spectra obtained for field directions $15^{\circ}$ from an element diagonal, a single peak was observed at a frequency similar to that of the mode observed with the field parallel to a diagonal.

In order to understand the observed behavior, we simulated the magnetization dynamics within both an isolated element and a model array of $3 \times 3$ identical elements so that the center element of the array had the same neighborhood as in the experiment. The simulations were performed with the parameter values determined experimentally in Ref. 23 and used in the simulations of Ref. 24. In order to avoid numerical artifacts due to the presence of sharp edges, ${ }^{27,28}$ the shape of the element was derived from a scanning electron microscope image of the array. The static state at each bias field value and orientation was prepared by allowing the magne- 


\section{Field Orientation}

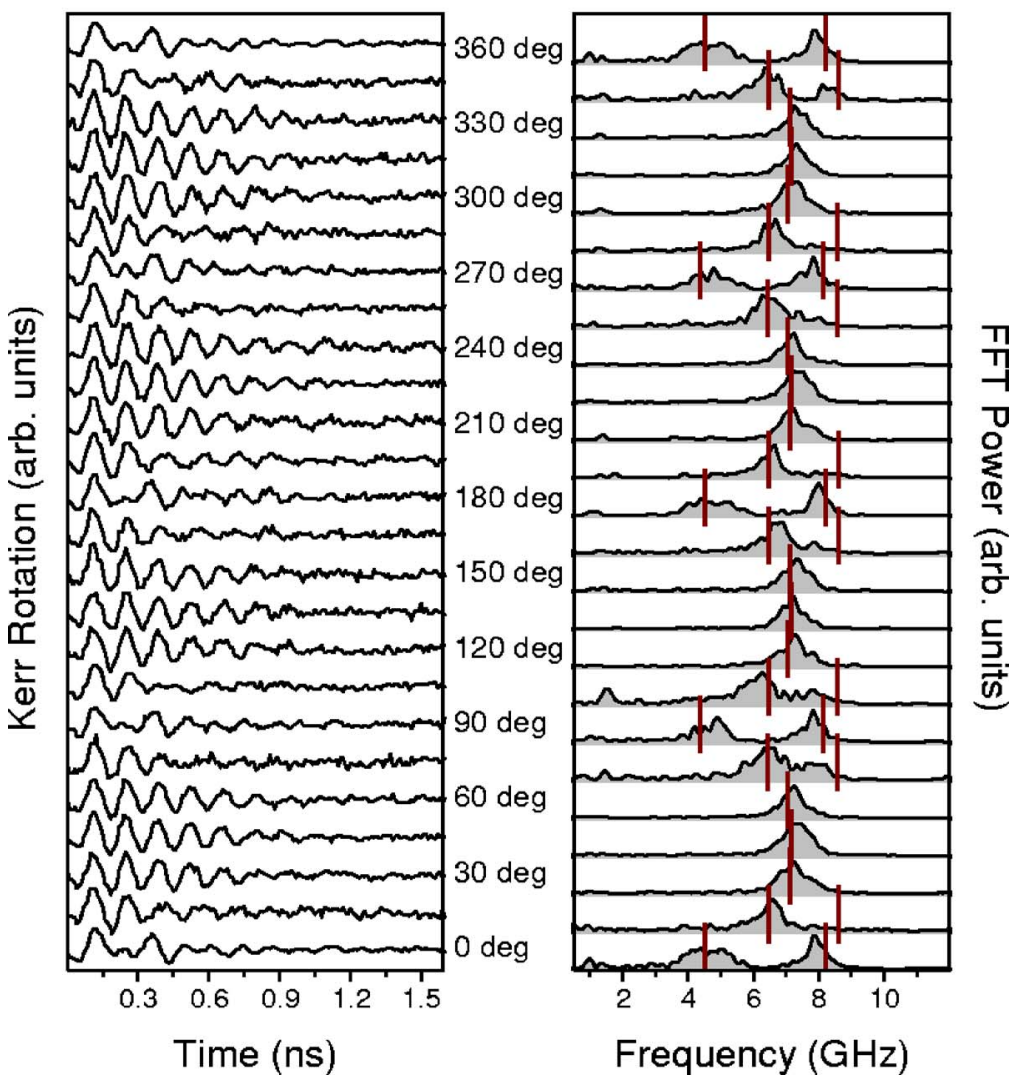

FIG. 2. (Color online) TR Kerr signals (left panel) and their FFT spectra (right panel) obtained for different orientations of the bias magnetic field are shown for the field value of 589 Oe. For $0^{\circ} / 360^{\circ}, 90^{\circ}, 180^{\circ}$, and $270^{\circ}$ the field lies parallel to an edge of the element. The vertical dashes show the frequencies of the modes obtained from the micromagnetic simulations. tization to relax from a uniform state. These static states were then used as the initial configuration in two kinds of dynamical simulation. In the first, the sample was excited by an out-of-plane pulsed field. ${ }^{24}$ In the second, a small harmonic out-of-plane field was applied with frequency corresponding to one of the modes identified from the response to the pulsed excitation. ${ }^{18,26}$

As indicated in Fig. 1 and 2, the simulations for the model array with a pulsed magnetic field reproduced well the ex- perimentally observed variation of the mode frequencies with the value and orientation of the bias magnetic field. Figure 3 compares the FFT spectra calculated from the outof-plane component of the dynamic magnetization averaged over the volume of the isolated element, and of the same element situated in the center of the model array in response to the pulsed excitation. The two simulations were in excellent agreement for all bias field orientations, except for a tiny difference of the edge mode frequency. Therefore we can

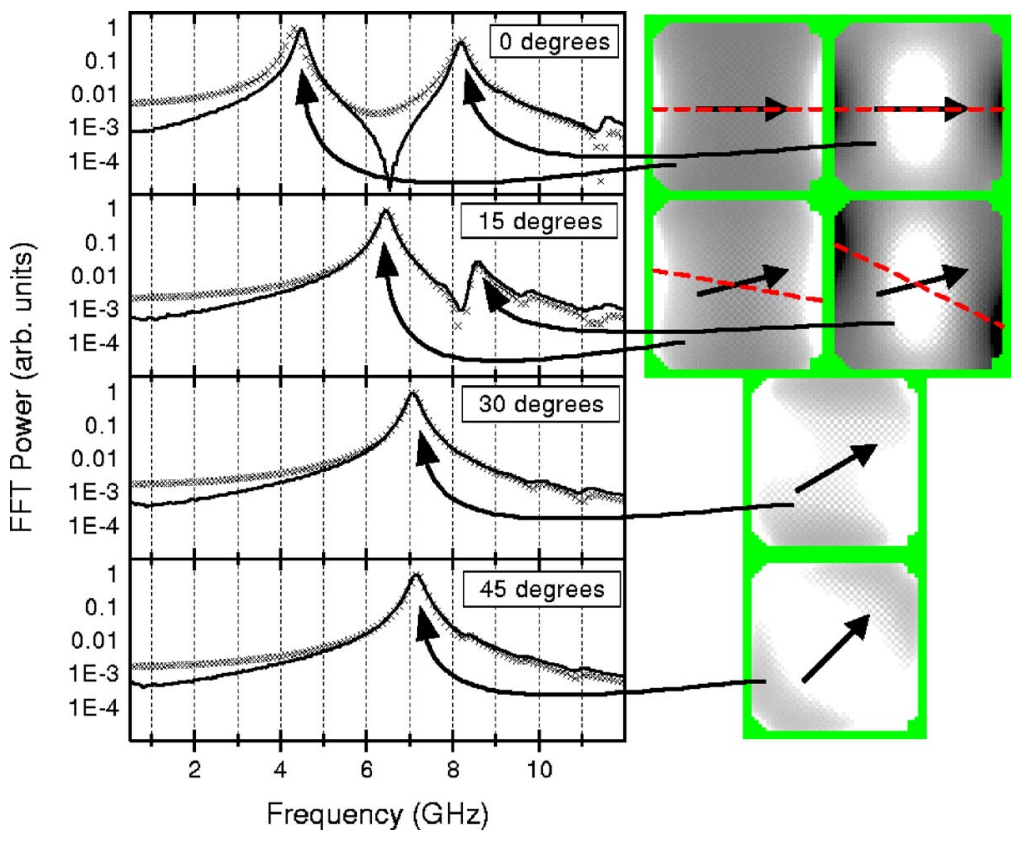

FIG. 3. (Color online) Simulated FFT spectra of the out-of-plane component of the average dynamic magnetization of the center element in a $3 \times 3$ array (solid line) and of an isolated element (symbols) in response to a pulsed magnetic field are shown for four different orientations of a bias field of 589 Oe. The dynamic configurations of the magnetization within an isolated element subject to a harmonic magnetic field at a frequency corresponding to the FFT peak marked by the arrow are shown in the images in the right column. White and black correspond to the positive and negative values of the dynamic out-of-plane component of magnetization, respectively. The arrows and the dashed lines in the images represent the directions of the bias field and of the effective wave vector, respectively. 


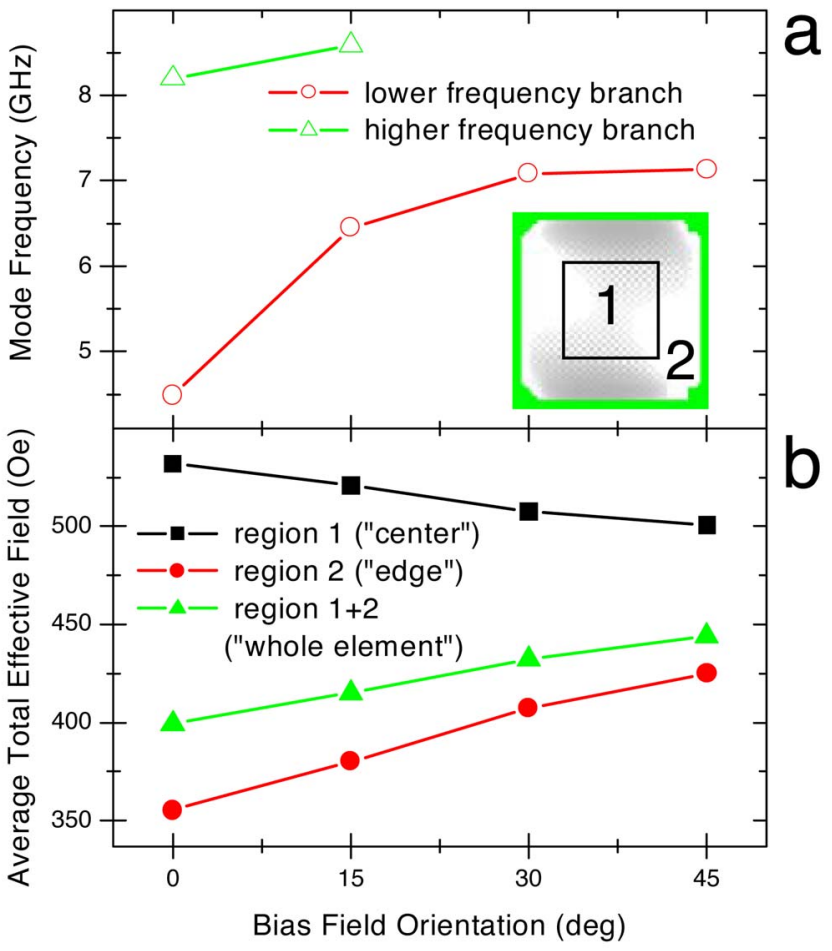

FIG. 4. (Color online) The dependence of the calculated mode frequencies (a) and of the total effective field averaged over different regions of the sample (b) upon the orientation of the bias magnetic field is shown. The inset shows the center (1) and edge (2) regions of the sample over which the averaging was performed.

neglect effects associated with the extrinsic configurational anisotropy in the present discussion. The images of the dynamic magnetization within the individual element in response to the harmonic excitation are shown in Fig. 3 as the bias field is rotated between the directions parallel to the edge and to the diagonal of the element.

The mode frequencies calculated from the simulations for the isolated element are plotted in Fig. 4(a) for different orientations of the bias magnetic field, while Fig. 4(b) shows the corresponding values of the static effective field averaged over the center and edge regions of the element, as well as the entire element. As expected from Ref. 5, because of the large aspect (size-to-thickness) ratio, the variation of the static effective field with the applied field angle due to the static configurational anisotropy $(<70 \mathrm{Oe})$ is too small, on its own, to explain the observed variation of the frequency of the lowest lying mode $(>2.5 \mathrm{GHz})$. Indeed, one can estimate from Fig. 1 that such a frequency change requires a field change of about 250 Oe. In additional simulations, the magnitude of the applied field for the different orientations was slightly adjusted (by less than $16 \mathrm{Oe}$ ) so that the value of the average static effective field was the same in each case. This resulted in a modification of the mode frequencies by less than $0.1 \mathrm{GHz}$. Therefore we conclude that the observed effects must be due to dynamic rather than static configurational anisotropy.

\section{DISCUSSION}

If the Hamiltonian describing a magnetic element is time invariant, the energy of small perturbations to the ground state (magnons) is conserved in the absence of the magnetic damping. The spin wave mode frequencies $f$ then represent "good" quantum numbers, and can be obtained by solving the following eigenvalue problem, derived from the linearized Landau-Lifshitz equation ${ }^{29}$

$$
\begin{aligned}
i 2 \pi f \mathbf{m}_{f}(\mathbf{r})= & -\gamma\left\lfloor\mathbf{m}_{f}(\mathbf{r}) \times \mathbf{H}_{\mathrm{E}}(\mathbf{H}, \mathbf{M}(\mathbf{H}, \mathbf{r}))\right\rfloor \\
& -\chi\left\lfloor\mathbf{M}(\mathbf{H}, \mathbf{r}) \times \mathbf{h}_{\mathrm{E}}\left(\mathbf{m}_{f}(\mathbf{r})\right)\right\rfloor,
\end{aligned}
$$

where $\mathbf{M}$ and $\mathbf{H}_{\mathrm{E}}$ are the static magnetization and static total effective field, $\gamma$ is the gyromagnetic ratio, and $\mathbf{r}$ is the spatial coordinate. The angular variation of $\mathbf{H}_{\mathrm{E}}$ at a specific location is responsible for the static configurational anisotropy. The dynamic effective field $\mathbf{h}_{\mathrm{E}}$ is related to the dynamic magnetization $\mathbf{m}_{f}$ for a particular mode by a linear integrodifferential operator, ${ }^{22}$ and is responsible for the dynamic configurational anisotropy.

The translational invariance of the system is broken in both in-plane directions due to the nonuniform static effective field and magnetization. ${ }^{24,26}$ Hence, it is impossible to ascribe a constant wave vector (momentum) to a particular mode. Moreover, a coordinate dependent wave vector, such as that introduced in Ref. 22 for a long stripe, is inappropriate here because it is impossible to assign a direction to the wave vector. However, in order to gain some insight into the mechanisms that underlie the observed variations in frequency, it is useful to make comparison with the dispersion of dipolar-exchange spin waves excited within a continuous ultrathin film i.e., in a system with translational invariance for which the in-plane wave vector is well defined. Neglecting any in-plane anisotropy, the frequency of the spin wave modes depends upon the angle $\varphi$ between the in-plane wave vector $(|\mathbf{k}|=k=2 \pi / \lambda)$ and the static magnetization as ${ }^{30}$

$$
2 \pi f(\varphi)=\gamma \sqrt{\left(H+\frac{2 A}{M} k^{2}+2 \pi M k d \sin ^{2} \varphi\right)\left[H+\frac{2 A}{M} k^{2}+4 \pi M\left(1-\frac{k d}{2}\right)\right]},
$$

where $A$ is the exchange constant and $M$ is the saturation magnetization. Let us now introduce an effective "wave vector direction" as shown in Fig. 3. This direction appears to lie parallel to the field when the field is applied along the edge of the element, but seems to be strongly rotated from the direction of the field when the field is applied at $15^{\circ}$ from the edge. Equation (2) shows that the dynamic dipolar interaction influences the frequency through both the magnitude of the wave vector (in the terms containing $k d$ ) and its direction $\varphi$ with respect to the static magnetization, while the exchange interaction 
influences the frequency only through the magnitude of the wave vector. Using the parameter values stated previously, Eq. (2) can easily account for the observed frequency variation if $\varphi$ and $k$ are allowed to vary by about $15^{\circ}$ and $10 \%$ respectively as the orientation of the applied field is varied.

The arguments presented in the previous paragraph may allow us to explain qualitatively the dynamic configurational anisotropy in terms of the dispersion of dipolar-exchange spin waves. A more rigorous analysis requires Eq. (1) to be solved in terms of a more appropriate set of eigenfunctions. ${ }^{22,31}$ However, the solution should also take into account the transition of the mode character from "edge" to "center" observed in Fig. 3 as the orientation of the bias field is changed. As the modes span different areas of the element, they also "see" different static effective magnetic fields and hence different contributions from the static configurational anisotropy, as indicated in Fig. 4. However, the frequency of the lowest mode changes only slightly at the mode crossover from the edge to center character observed at the field orientations of $30^{\circ}$ and $45^{\circ}$. This suggests that, in the present case, changes in the effective wave vector drive the variation in frequency of the lowest lying mode as the orientation of the static field is changed. Although the regions over which the static field is averaged in Fig. 4 provide only a crude approximation of the regions of confinement of the edge and center modes, they give a good qualitative account of the small variation of the static effective field. A rigorous analytical theory of the observed phenomena has yet to be developed and is beyond the scope of this experimental paper.

\section{CONCLUSIONS}

In summary, the resonant modes of a square shaped magnetic nanoelement have been studied experimentally and numerically. Both the number of modes and their frequencies were observed to vary as the orientation of the external magnetic field was rotated in the plane of the element. We have shown that the observed variation cannot be explained by either the effect of the extrinsic configurational anisotropy or the static intrinsic configurational anisotropy, but is instead due to the variation of the dynamic effective field - a $d y$ namic intrinsic configurational anisotropy. This new anisotropy can dominate the ultrafast magnetization dynamics of ultrathin nanomagnets for which the static intrinsic configurational anisotropy is of minor importance.

\section{ACKNOWLEDGMENTS}

The authors acknowledge the financial support of the UK Engineering and Physical Sciences Research Council (EPSRC) and the New Energy and Industrial Technology Development Organization (NEDO).
${ }^{1}$ E. Noether, Nachr. Ges. Wiss. Goettingen, Math.-Phys. Kl. 2, 235 (1918).

${ }^{2}$ Ultrathin Magnetic Structures I, edited by B. Heinrich and J. A. C. Bland (Springer-Verlag, Berlin, 1994).

${ }^{3}$ A. Hubert and R. Schäfer, Magnetic Domains: the Analysis of Magnetic Microstructures (Springer-Verlag, Berlin, 1998).

${ }^{4}$ M. E. Schabes and H. N. Bertram, J. Appl. Phys. 64, 1347 (1988)

${ }^{5}$ R. P. Cowburn and M. E. Welland, Phys. Rev. B 58, 9217 (1998).

${ }^{6}$ R. P. Cowburn, A. O. Adeyeye, and M. E. Welland, Phys. Rev. Lett. 81, 5414 (1998).

${ }^{7}$ R. P. Cowburn, D. K. Koltsov, A. O. Adeyeye, and M. E. Welland, Europhys. Lett. 48, 221 (1999).

${ }^{8}$ S. M. Chérif, C. Dugautier, J.-F. Hennequin, and P. Moch, J. Magn. Magn. Mater. 175, 228 (1997).

${ }^{9}$ S. M. Chérif, Y. Rousigné, C. Dugautier, and P. Moch, J. Magn. Magn. Mater. 242-245, 591 (2002).

${ }^{10}$ Y. Zhai, J. Shi, X. Y. Zhang, L. Shi, Y. X. Xu, H. B. Haung, Z. H. Lu, and H. R. Zhai, J. Phys.: Condens. Matter 14, 7865 (2002).

${ }^{11}$ M. Pardavi-Horvath, C. A. Ross, and R. D. McMichael, IEEE Trans. Magn. 41, 3601 (2005).

${ }^{12}$ A. Barman, V. V. Kruglyak, R. J. Hicken, A. Kundrotaite, and M. Rahman, Appl. Phys. Lett. 82, 3065 (2003).

${ }^{13}$ A. Barman, V. V. Kruglyak, R. J. Hicken, A. Kundrotaite, and M. Rahman, J. Magn. Magn. Mater. 272-276, 2121 (2004).

${ }^{14}$ A. Barman, V. V. Kruglyak, R. J. Hicken, J. Scott, A. Kundrotaite, and M. Rahman, J. Appl. Phys. 95, 6998 (2004).

${ }^{15}$ A. Barman, V. V. Kruglyak, R. J. Hicken, J. M. Rowe, A. Kun- drotaite, J. Scott, and M. Rahman, Phys. Rev. B 69, 174426 (2004).

${ }^{16}$ C. Mathieu, C. Hartmann, M. Bauer, O. Buettner, S. Riedling, B. Roos, S. O. Demokritov, B. Hillebrands, B. Bartenlian, C. Chappert, D. Decanini, F. Rousseaux, E. Cambril, A. Müller, B. Hoffmann, and U. Hartmann, Appl. Phys. Lett. 70, 2912 (1997).

${ }^{17}$ B. Hillebrands, C. Mathieu, C. Hartmann, M. Bauer, O. Büttner, S. Riedling, B. Roos, S. O. Demokritov, B. Bartenlian, C. Chappert, D. Decanini, F. Rousseaux, E. Cambril, A. Müller, B. Hoffmann, and U. Hartmann, J. Magn. Magn. Mater. 175, 10 (1997).

${ }^{18}$ S. Jung, B. Watkins, L. DeLong, J. B. Ketterson, and V. Chandrasekhar, Phys. Rev. B 66, 132401 (2002).

${ }^{19}$ G. N. Kakazei, P. E. Wigen, K. Y. Guslienko, R. W. Chantrell, N. A. Lesnik, V. Metlushko, H. Shima, K. Fukamichi, Y. Otani, and V. Novosad, J. Appl. Phys. 93, 8418 (2003).

${ }^{20}$ G. N. Kakazei, P. E. Wigen, K. Y. Guslienko, V. Novosad, A. N. Slavin, V. O. Golub, N. A. Lesnik, and Y. Otani, Appl. Phys. Lett. 85, 443 (2004).

${ }^{21}$ R. W. Damon and J. R. Eshbach, J. Phys. Chem. Solids 19, 308 (1961).

${ }^{22}$ C. Bayer, J. P. Park, H. Wang, M. Yan, C. E. Campbell, and P. A. Crowell, Phys. Rev. B 69, 134401 (2004).

${ }^{23}$ V. V. Kruglyak, A. Barman, R. J. Hicken, J. R. Childress, and J. A. Katine, J. Appl. Phys. 97, 10A706 (2005).

${ }^{24}$ V. V. Kruglyak, A. Barman, R. J. Hicken, J. R. Childress, and J. A. Katine, Phys. Rev. B 71, 220409(R) (2005).

${ }^{25}$ M. Donahue and D. G. Porter, OOMMF User's guide, Version 1.0, Interagency Report NISTIR 6376, NIST, Gaithersburg, MD, 
1999.

${ }^{26}$ V. V. Kruglyak, P. S. Keatley, R. J. Hicken, J. R. Childress, and J. A. Katine, J. Appl. Phys. 99, 08F306 (2006).

${ }^{27}$ A. Aharoni, J. Appl. Phys. 83, 3432 (1998).

${ }^{28}$ A. Aharoni, Physica B 306, 1 (2001).

${ }^{29}$ A. G. Gurevich and G. A. Melkov, Magnetization oscillations and waves (CRC Press, Boca Raton, 1996).

${ }^{30}$ J. F. Cochran, J. Rudd, W. B. Muir, B. Heinrich, and Z. Celinski, Phys. Rev. B 42, 508 (1990).

${ }^{31}$ C. Bayer, J. Jorzick, B. Hillebrands, S. O. Demokritov, R. Kouba, R. Bozinoski, A. N. Slavin, K. Y. Guslienko, D. V. Berkov, N. L. Gorn, and M. P. Kostylev, Phys. Rev. B 72, 064427 (2005). 